\title{
Research Status of Automatic Train Operation for Metro
}

\author{
Liu Xiaohan, Shen Hanlin \\ Department of electrical engineering, Aviation engineering institute, Civil Aviation Flight University of \\ China. China \\ liuxioahancai@126.com
}

\section{Keywords: Automatic Train Operation; Communication-Based Train Control; Metro}

\begin{abstract}
This paper investigates the present development situation and summarizes the current control algorithm and the technical difficulties of Automatic Train Operation for Metro. Based on the analysis of the current Automatic Train Operation, the combination of offline optimization and on-line adaptive control will be the development priorities. And it raises a number of issues that must be focused on of Automatic Train Operation.
\end{abstract}

\section{Introduction}

Train operation control is a typical non-linear multi-objective optimization problem. Automatic Train Operation (ATO) has a great advantage over manned train operation in operating efficiency. And it is in line with the development trend of information and intelligence especially in the metro field. The characteristics of metro are a greater tracking density, frequent starting and braking, a relatively simple road network structure and relatively fixed operating environment to railway. It can be concluded that metro is high request on the parking accuracy, punctuality, energy performance and it is less interfered and easy to control. ATO adopts the advanced automatic control technology and it greatly improves the driving efficiency and safety which determines the rapidly developing of ATO in metro.

The metro almost adopt the advanced Communication-Based Train Control (CBTC) in recent years. CBTC uses a reliable, high-bandwidth wireless communication to replace the track circuit, transponder to achieve the intercommunication between the train and the ground equipment which provides the possibility to achieve the precise control of train. The total length of metro which go into operation by the end of 2013 is around $2305 \mathrm{~km}$ in China shown as Figure 1. Almost $80 \%$ of them use $\mathrm{ATO}^{[1]}$ which is imported from Siemens, Alstom, Bombardier, Ansaldo and other companies. So the study of ATO has direct economic and social benefits.

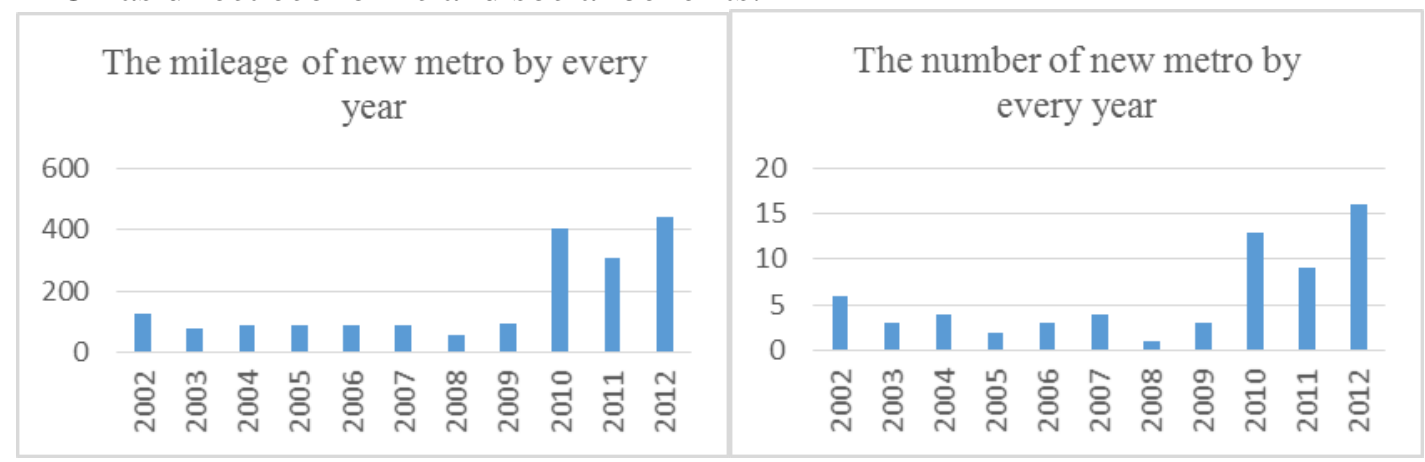

Figure 1. The length of metro in China

\section{ATO}

ATO is the core and the decision center of CTCS. ATO makes use of basis information as line conditions, basis parameters of train, train dynamic performance, etc and dynamic information as the speed, position, acceleration, over-speed protection, temporary speed restriction, etc to calculate and output the current operation conditions and control level of the train in real-time to automatically control the train ${ }^{[2]}$. The evaluation of ATO's control performance may be slightly different for 
different operation environment. The performance indexes of metro ATO are safety, punctuality, parking precision, energy consumption and comfort. But theses indexes cannot be met simultaneously such as punctuality and energy consumption. The lower the speed is, the lower the energy consumption is, but the low speed is not conductive to punctuality. So it need to be coordinated when studying the control algorithm of ATO to achieve satisfactory control effect.

\section{Control algorithm and technical difficulties of ATO}

The essence of ATO is to solve the train operating conditions (position, handle level) and its core is the design and implementation of optimizing control algorithm. The present research mainly concentrates on two parts. One is to solve the offline operation optimization curve of ATO, the other one is the online control and real-time adjustment of ATO. The present research focus on the combination of offline operation optimization curve and real-time adjustment.

\subsection{Offline operation optimization curve}

The offline operation optimization curve refers to the curve which is simulated and calculated under the specific traction calculation model and external constraints. The offline operation optimization curve contains the information about all operation conditions of the train.

Since the running time of the train is not fixed, the timetable may be adjusted at any time, it can be required to generate multiple optimization curve according to different running time. These curves can be cured in the control system of the train, and the train can generate the corresponding control instruction based on the operation conditions according the cures to control the train. As Figure 2 shows that multiple offline operation optimization curves are cured in ATO, when the timetable changes, the on-board controller will cheese different optimization curves as the reference to control train operation.

The offline operation optimization curve is not suitable for online and real-time calculation and adjustment as its generation needs large amount of calculation which sues subsection solution according to different operation phase, and takes the line conditions and energy saving operation principles into consideration. The offline operation optimization curve can only be calculated from fixed train quality which may change because of the number of passengers and the train road which leads the offline operation optimization curve is not completely consistent with the actual conditions.

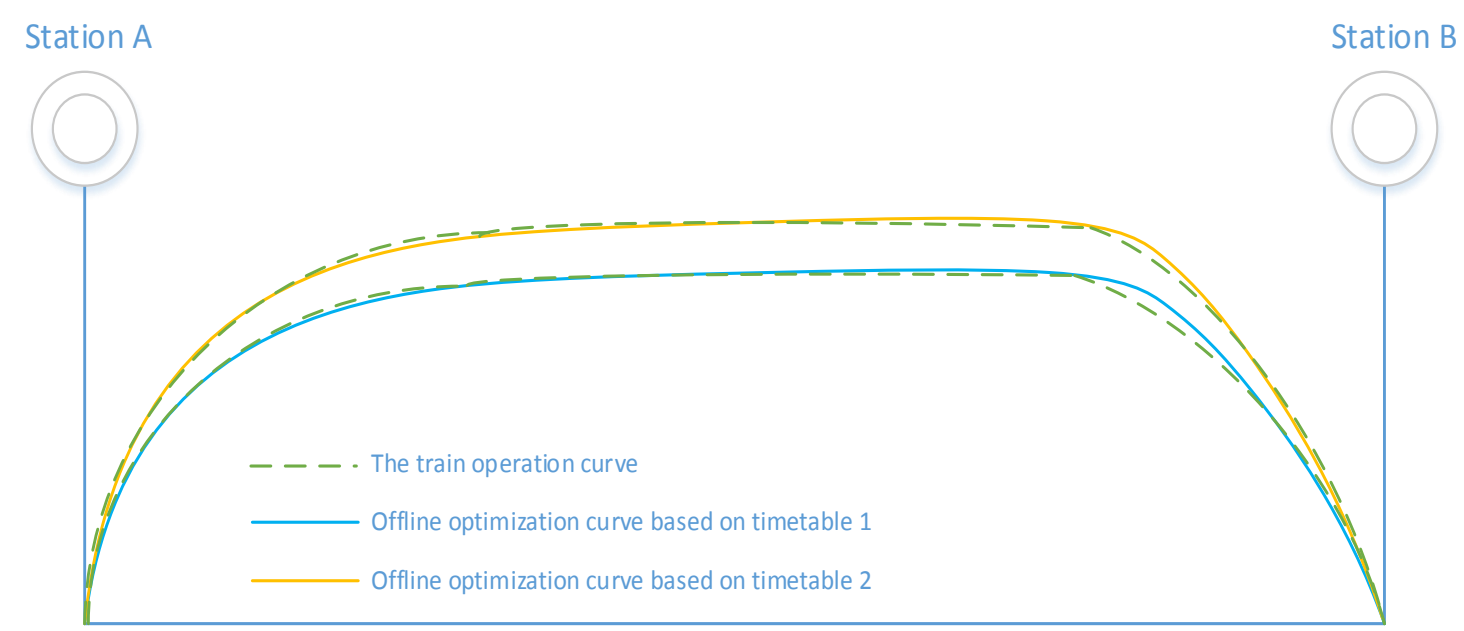

Figure2. The offline operation optimization curve

\subsection{Online operation control}

Online operation control means that when the train quality, resistance and the model parameters change, ATO can adjust operation control to achieve optimal efficiency. In the offline optimization, ATO will pre-store several offline operation optimization curves to correspond to different running time which is usually far greater than the maximum running time. So when making the timetable, it will reserve some time margin which provides the possibility for the online operation control of ATO. 
The processes of online operation control are as follows: when there is offset between running time and timetable, ATO will determine which curve the train should be in accordance with according to offset and the current position.

Online operation control relies on the offline operation optimization curve. The advantage of online operation is that it can adjust the operation control quickly and timely to ensure the punctuality and energy-saving operation. The offline operation optimization curve is pre-set and it can only correspond to discrete time. When train delay or timetable changing, it can only be back to rough running time. So the online operation control is lack of control accuracy and poor adaptability. Figure 3 is the online adjustment curve of ATO.

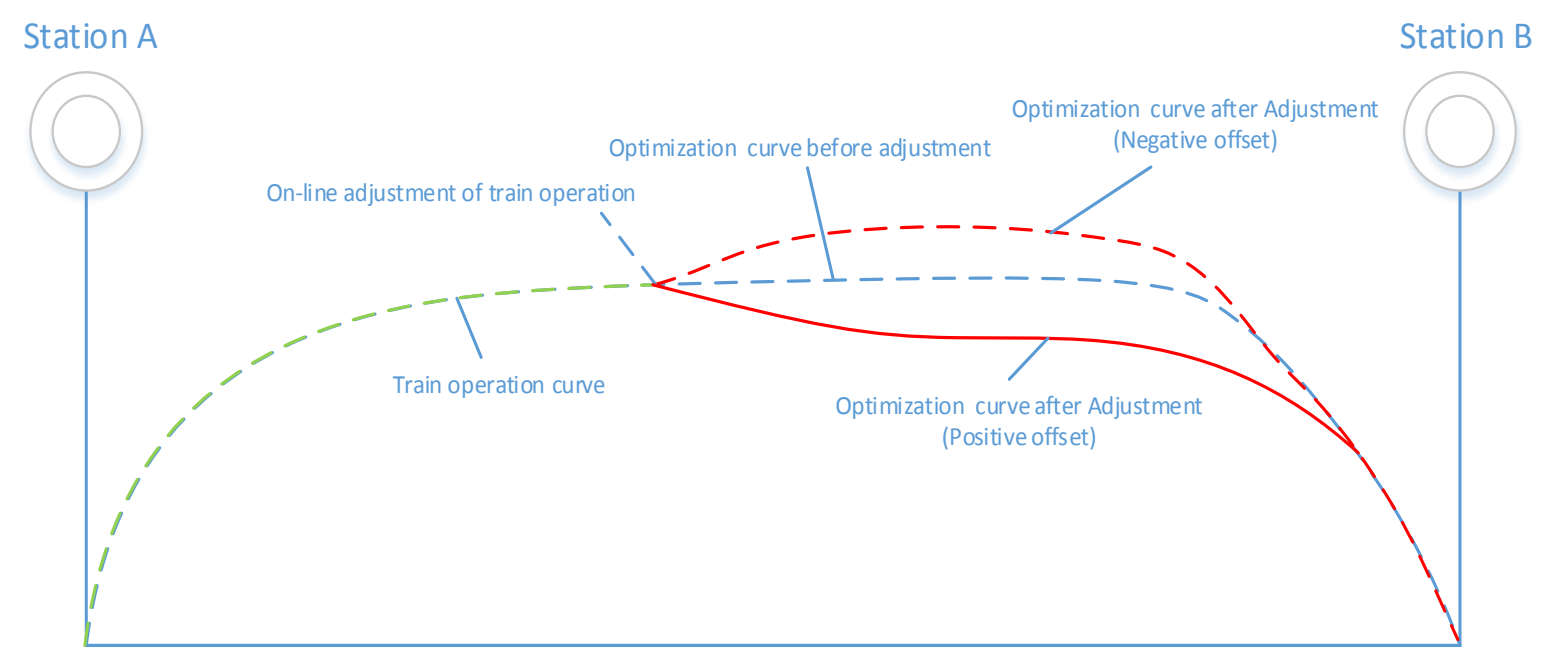

Figure3. Online adjustment curve of ATO

As general, the cause of the timetable offset can be summarized in two situation.

One is the train delay. There are many possible reasons for the train delay. The conflict and interference of train tracking is the main reason that causes the train delay in the simulation. And the timetable offset can be calculated by the train's current position running time $t_{i}$ and the offline operation optimization curve's current position running time $t_{\text {opti }}$.

$$
\Delta t=t_{i}-t_{\text {opti }}
$$

The other one is the train running interval adjustment by ATS. If the ATS adjust the current running time interval, and the other running interval need to be adjusted accordingly. The train's current position running time is $t_{i}$, original set interval running time is $T_{i}^{\prime}$, set interval running time after adjusting is $T_{i}$.

$$
\Delta t=\left(T_{i}-t_{i}\right)-\left(T_{i}^{\prime}-t_{i}\right)
$$

When the time offset exceeds a certain range, ATO will adjust to select the offline optimization curve based on the current position of the train and offset.

\section{Simulation}

The simulation is based on VC++, and takes the Shanghai-Nanjing high-speed rail line and CRH2 for example. The start and destination of the line are marked by $360049 \mathrm{~m}$ and $381136 \mathrm{~m}$. The single-particle traction model is adopted and the simulation of speed-distance curve is shown as Figure 4. 


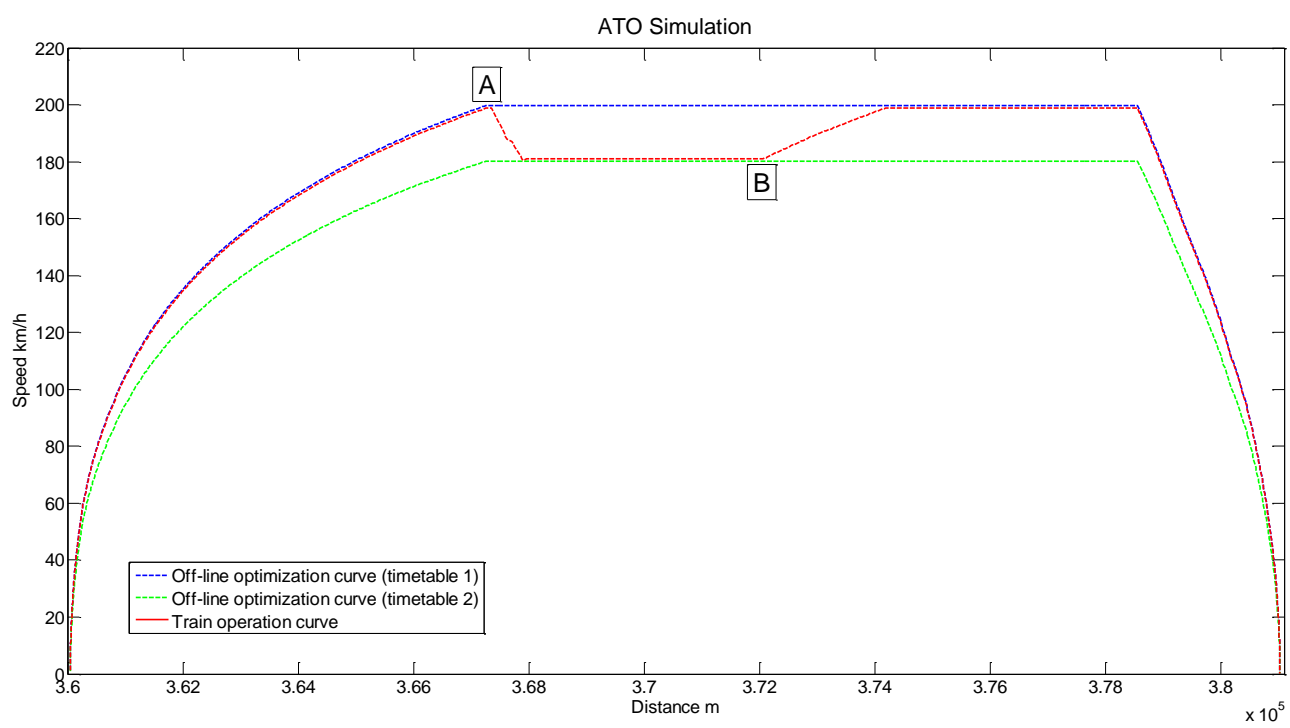

Figure 4. The speed-distance curves of ATO

This simulation is based on two offline operation optimization curves which one correspond to 8 ' 20 ' of timetable1 and the other one correspond to 9'7'' of timetable2 and combines with online operation control. The initial interval running time of the train is 8 ' 20 '. In the start-up stage, since timetable 1 is close to the requirement interval running time, ATO will choose the offline operation optimization curve that corresponded to timetable 1 to control the train. When the train arrives at the position A, shown as Figure 4, the time offset is +40s and ATO will choose the offline operation optimization curve that corresponded to timetable 2 according to the present position of the train and the time offset to control the train. When the train arrives at the position $\mathrm{B}$, the time offset is negative, ATO will choose the curve that corresponded to timetable1.

The acceleration curve is shown as Figure 5and the operation curve of handle is shown as Figure 6 which reflect the two adjustment processes of ATO. In parking stage, ATO take the precise stop as the goal and control the handle level and the acceleration changing frequently.

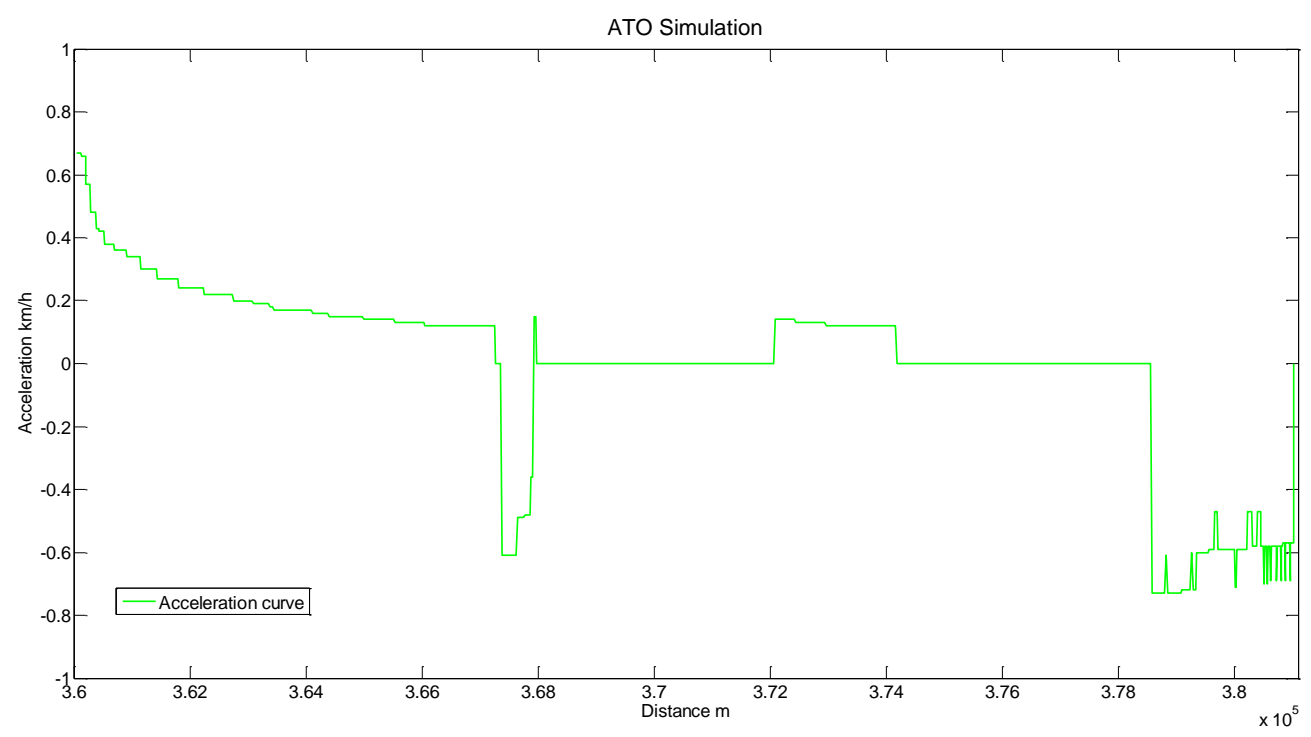

Figure 5. The acceleration curve 


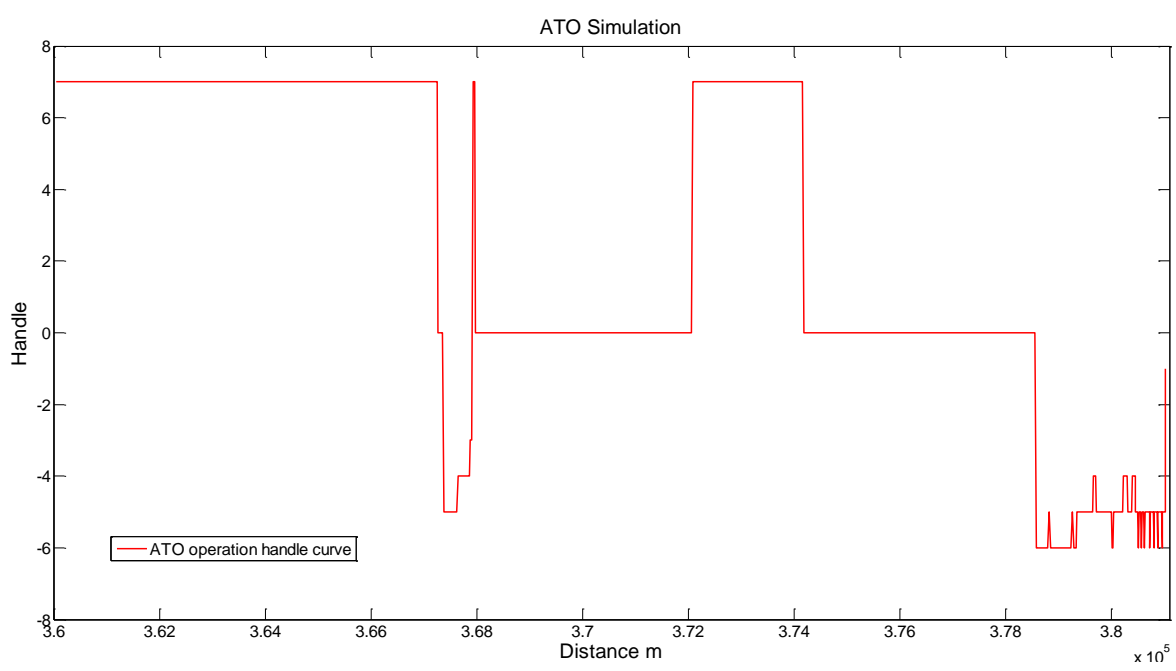

Figure 6. The operation handle curve

The control index of ATO is shown as Table 1.

Table 1. Control index of ATO

\begin{tabular}{cccc}
\hline Index & Expected value & Actual value & Error \\
\hline Stop accuracy & $381026 \mathrm{~m}$ & $381025.94 \mathrm{~m}$ & $-0.06 \mathrm{~m}$ \\
Punctuality rate & $8^{\prime} 20^{\prime}$, & $89^{\prime}$, & $+9 \mathrm{~s}$ \\
\hline
\end{tabular}

\section{Existing problems of ATO}

ATO has been applied currently, but there are also many problems such as the technical difficulties. ATO also affected by many external factors. Transmission efficiency of the train in impossible to reach 1 , it changes with the speed, when designing the ATO system, it should be taken into consideration.

\section{References}

[1] Tang Tao and Liangji Huang 2003 Automatic train operation algorithm summary. Journal of railway. 225

[2] Changlin Wang and Lin Yin 2008 Train operation control technology (ChengDu: Southwest Jiaotong University) pp 10-22

[3] Chang C 1997 Optimizing Train Movements through Coast Control Using Genetic Algorithms. IEE Proc. -Electr.PowerAppl. 144 pp 65-73

[4] Chang C and Sim S 1999 Pareto-optimal Set Based Multi objective Tuning of Fuzzy Automatic Train Operation for Mass Transit System. IEE Proc. -Electr. Power Appl.146

[5] Eugene K 2000 On an Optimal Control Problem of Train Operation. IEE Proc. -Electr. Power Appl. 457

[6] Yasunobu S 1984 Application of Predictive Fuzzy Control to Automatic Train Operation Controller. In:Proc.of IECON 84 